\title{
Challenges and Opportunities for Extracting Cardiovascular Risk Biomarkers from Imaging Data
}

\author{
I.A. Kakadiaris ${ }^{1}$, E.G. Mendizabal-Ruiz ${ }^{1}$, U. Kurkure $^{1}$, and M. Naghavi ${ }^{2}$ \\ 1 Computational Biomedicine Lab, Departments of Computer Science, \\ Electrical and Computer Engineering, and Biomedical Engineering \\ University of Houston, Houston, TX \\ 2 Society for Heart Attack Prevention and Eradication (SHAPE), Houston, TX \\ http://www.cbl.uh.edu
}

\begin{abstract}
Complications attributed to cardiovascular diseases (CDV) are the leading cause of death worldwide. In the United States, sudden heart attack remains the number one cause of death and accounts for the majority of the $\$ 280$ billion burden of cardiovascular diseases. In spite of the advancements in cardiovascular imaging techniques, the rate of deaths due to unpredicted heart attack remains high. Thus, novel computational tools are of critical need, in order to mine quantitative parameters from the imaging data for early detection of persons with a high likelihood of developing a heart attack in the near future (vulnerable patients). In this paper, we present our progress in the research of computational methods for the extraction of cardiovascular risk biomarkers from cardiovascular imaging data. In particular, we focus on the methods developed for the analysis of intravascular ultrasound (IVUS) data.
\end{abstract}

Keywords: vulnerable patients, intravascular ultrasound, vasa vasorum.

\section{Introduction}

The complications attributed to cardiovascular diseases (CDV) are the leading cause of death worldwide. For a significant percentage of patients, the first symptom of CVD is sudden death without previous warnings. In the United States, sudden heart attack remains the number one cause of death and accounts for the majority of the $\$ 280$ billion burden of cardiovascular diseases. Cardiovascular specialists indicate that heart attacks are caused by inflammation of the coronary arteries and thrombotic complications of vulnerable plaques. The concept of vulnerable plaque has recently evolved into the definition of "vulnerable patient". A vulnerable patient is defined as a person with more than a $10 \%$ likelihood of having a heart attack in the next 12 months. Over 45 world leaders in cardiology have collectively introduced the field of vulnerable patient detection as defining the new era in preventive cardiology [1].

E. Bayro-Corrochano and J.-O. Eklundh (Eds.): CIARP 2009, LNCS 5856, pp. 227-235, 2009.

(C) Springer-Verlag Berlin Heidelberg 2009 
Detection of vulnerable patients is one of the most active areas of research in both the cardiology and biomedical imaging communities. While there exist many invasive and non-invasive medical imaging modalities for the study and diagnosis of CVD, until now none of them can completely identify a vulnerable plaque and accurately predict its further development. Therefore, the necessity of novel methods for extracting cardiovascular risk biomarkers from these image modalities is evident.

Cardiovascular risk biomarkers. The presence of calcified coronary plaque has a significant predictive value for coronary artery disease and is associated with with cardiovascular risk [2,345]. Furthermore, vasa vasorum (VV) neovascularization on the plaque has been identified as a common feature of inflammation [6] and has been defined as a plaque vulnerability index. Other cardiovascular risk biomarkers include calcification in the aorta and thoracic fat burden.

Our group is pioneering work in the development of computational methods for mining of information from different modalities of invasive and non-invasive cardiovascular imaging data. For the non-invasive image data analysis, using cardiac CT data, we have presented methods for the automatic detection of coronary artery regions 78, automatic detection of coronary calcium using a hierarchical supervised learning framework 91011, automatic delineation of the inner thoracic region [12, and segmentation of thoracic aorta [13. Additionally, the thoracic fat is detected using a relaxed version of multi-class, multi-feature fuzzy connectedness method [14].

Furthermore, our group has developed several computational methods for the analysis of intravascular ultrasound (IVUS) contrast imaging for the detection of VV in-vivo [1516. These methods include techniques for IVUS image stabilization and differential imaging techniques for the detection of those changes which occur in IVUS imagery due to the perfusion of an intravascularly-injected contrast agent into the plaque and vessel wall. However, one of the limitations of these methods is related to the use of the Cartesian B-mode representation of the IVUS signal. This is a disadvantage because the transformation of the ultrasound radio frequency $(\mathrm{RF})$ signal data into this representation results in loss of potentially valuable information.

In this paper, we present ongoing work by our group in order to overcome these limitations and take full advantage of the information contained in the "raw" RF signal.

\section{Methods}

IVUS is currently the gold-standard technique for assessing the morphology of blood vessels and atherosclerotic plaques in vivo. The IVUS catheter consists of either a solid-state or a mechanically-rotated transducer which transmits a pulse and receives an acoustic signal at a discrete set of angles over each radial scan. Commonly, 240 to 360 such signals are obtained per (digital or mechanical) 
rotation. The envelopes of these signals are computed, log-compressed, and then geometrically transformed to obtain the familiar disc-shaped IVUS image.

IVUS has been combined with contrast-enhancing agents as blood tracers for the detection of blood perfusion due to VV 17181920]. The protocol for blood perfusion detection consists of injecting high-echogenic microbubbles of size similar to red blood cells into the blood flow while monitoring with IVUS. If these microbubbles are found beyond the lumen border, this could be an indication of microcirculation due to $\mathrm{VV}$.

\subsection{Contrast Agent Detection}

Based on this protocol, we have investigated the feasibility of detecting microbubbles in IVUS data by acoustic characterization of the raw RF IVUS data using two approaches based on one-class cost-sensitive learning [21. In the first approach, we built a model for the microbubbles from samples of microbubbles present in the lumen during the contrast agent injection. In the second approach, we detected the microbubbles as a change from baseline IVUS data consisting of samples of different tissues of the vessel extracted from frames before the injection.

For our models, we used those features based on frequency-domain spectral characterization that represent measures of high-frequency signal proposed by O'Malley et al. [16. Specifically, these features are defined for a 3-D signal window of dimensions $r_{0} \times \theta_{0} \times t_{0}$ as follows:

$$
\begin{aligned}
F_{\zeta} & =\sum_{i=1}^{\left\lceil r_{0} / 2\right\rceil} \sum_{j=1}^{\left\lceil\theta_{0} / 2\right\rceil} \sum_{k=1}^{\left\lceil t_{0} / 2\right\rceil} i j k \hat{W}(i, j, k) \\
F_{\eta} & =\frac{F_{\zeta}}{\sum_{i=1}^{\left\lceil r_{0} / 2\right\rceil} \sum_{j=1}^{\left\lceil\theta_{0} / 2\right\rceil} \sum_{k=1}^{\left\lceil t_{0} / 2\right\rceil} \hat{W}(i, j, k)},
\end{aligned}
$$

where $\hat{W}$ indicates the magnitude of the Fourier spectrum of the windowed signal $W$. Each feature is computed on $I_{e}$ and $I_{l}$ in addition to $I$. Hence, each feature is a vector of three values. The samples are extracted by placing a 3-D fixed size window $\left(r_{0}, \theta_{0}, t_{0}\right)$ around each sample in the volume. These features are computed for this window and associated with the class contained by it. To improve the scaling of the feature space, each feature of the samples used for training is normalized to zero mean and unit variance. The normalization values are retained for use in testing and deployment. The parameters of the one-class SVM $\gamma$ and $\nu$ are selected in such a way that good performance on the recognition of the class of importance and on the rejection of the negative class is obtained. However, since it is possible to have higher accuracy on the classification of negative samples than in the class of interest, we constrain the selection of parameters to provide an accuracy on the class of interest as close to $100 \%$ as possible. Therefore, the criteria for the selection of the best parameters is given by a weighted linear combination of the accuracy on the classification of both classes, $A=w_{1} A_{P}+w_{2} A_{N}$, where $A$ stands for total accuracy, $A_{P}$ and $A_{N}$ 
are the accuracies of detecting the class of interest and rejecting the negative class, respectively, and $w_{1}$ and $w_{2} \in[0,1]$ are the weights associated with the class of interest and negative class accuracy, respectively. This can be considered cost-sensitive learning for one-class classifiers.

\subsection{Scattering Model-Based Analysis of IVUS}

Currently, we are investigating the feasibility of using a physics-based scattering model of the IVUS RF signal for the analysis of the IVUS data. This model assumes that the IVUS signal can be obtained from a physical model based on the transmission and reflection of ultrasound waves that radially penetrate the arterial structure. Since the wavelength produced by IVUS transducers is very large in comparison to the dimension of the structures of the vessel, this model assumes that structures can be modeled as a finite set of point scatterers with an associated differential backscattering cross-section coefficient (DBC). In this model, the ultrasound beam interacts with scatterers along its radial direction along an angular window given by $\Delta \Theta=\sin ^{-1}\left(1.22 \frac{\lambda}{D}\right)$ (Fig. 1(a), where $\lambda=\frac{c}{f}$ is the wavelength, $f$ is the transducer frequency and $D$ is the transducer diameter. Assuming Born approximation scattering, we use the principle of superposition to represent the total scattered wave as a sum of reflections from individual point scatterers 22. Then, using this model, the ultrasound reflected signal for each transducer's angular position $\Theta_{k}$ at time $t$ for a finite set of $N$ scatterers with coordinates $\left(r_{i}, \theta_{i}\right)$ where $\theta_{i} \in\left\{\Theta_{k}-\frac{\Delta \Theta}{2}, \Theta_{k}+\frac{\Delta \Theta}{2}\right\}$ and DBC $\kappa\left(r_{i}, \theta_{i}\right)$ is given by:

$$
\hat{S}\left(t, \Theta_{k}\right)=\frac{1}{N} \sum_{i=1}^{N} \frac{\kappa\left(r_{i}, \theta_{i}\right) \exp \left(-\mu r_{i}\right)}{r_{i}} \exp \left(\frac{-\left(t-\frac{2 r_{i}}{c}\right)^{2}}{2 \sigma^{2}}\right) \sin \left(\omega t-\frac{2 r_{i}}{c}\right),
$$

where $\mu$ is the attenuation coefficient, $C$ defines the transducer constant parameters, and $\omega=2 \pi f$ is the angular velocity of the impulse function with width $\sigma$.

In order to be able to use this model, first it is necessary to recover its parameters. We accomplish this by solving an inverse problem on which we tune the parameters by the minimization of the difference between a ground truth signal and our modeled signal.

A significant difficulty is that the modeled signal depends on the position of the scatterers. We cannot treat the distribution of scatterers in a deterministic fashion: the scatterers' positions are the result of a spatial stochastic point process. Therefore, the minimization of the differences of the signals should be approached in a stochastic sense. We consider the optimal parameter values as functions of the scatterer locations. Then, for each angle $k$ we generate $\xi$ samplings of scatterer positions and minimize the sum of the errors between the real IVUS signal and each of the $\xi$ modeled signals. Specifically, we solve the problem: 


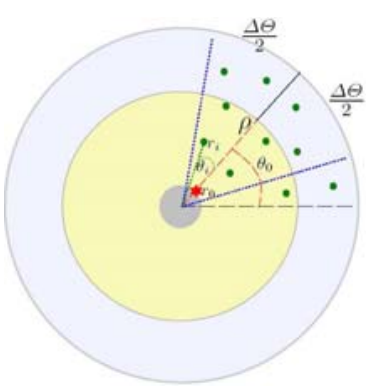

(a)

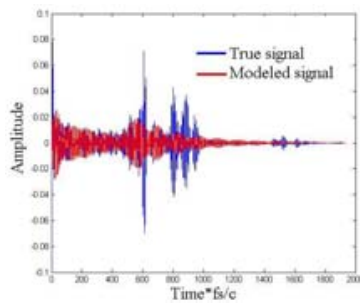

(b)

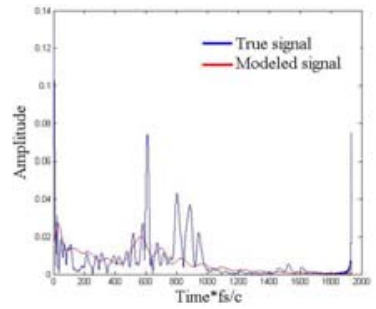

(c)

Fig. 1. (a) Scatterers interacting with the ultrasound beam on IVUS. (b) Raw real and modeled IVUS signals for a single angle. (c) Positive envelope of real and modeled IVUS signals for a single angle.

$$
\min _{\sigma_{k}, \kappa_{k}^{l}, \kappa_{k}^{w}} \frac{1}{2} \sum_{t} \sum_{i=1}^{\xi}\left(E\left(t, \Theta_{k}\right)-\hat{E}_{i}\left(t, \Theta_{k}, \sigma, \kappa^{l}, \kappa^{w}\right)\right)^{2},
$$

where $E\left(t, \Theta_{k}\right)$ and $\hat{E}_{i}\left(t, \Theta_{k}, \sigma, \kappa^{l}, \kappa^{w}\right)$ are the positive envelopes for the real and the modeled signals, respectively (Figs. 1(b) and 1(c)).

We have applied this model to the segmentation of the luminal border using the IVUS RF data [23]. For this, we consider that the radial position $\rho_{k}$ of the lumen border for each angle $\Theta_{k}$ can be recovered by solving an inverse problem as well. We use the parameters computed on the calibration and we find $\rho_{k}$ by the minimization of the sum of differences between the real IVUS signal $S\left(t, \Theta_{k}\right)$ and the signals computed with our model $\hat{S}_{i}\left(t, \Theta_{k}, \rho_{k}\right)$ for each sampling $\xi$. Specifically, we solve:

$$
\min _{\rho_{k}} \frac{1}{2} \sum_{t} \sum_{i=1}^{\xi}\left(E\left(t, \Theta_{k}\right)-\hat{E}_{i}\left(t, \Theta_{k}, \rho_{k}\right)\right)^{2} .
$$

\section{Results}

Regarding the detection of contrast agent, for the first approach, we obtained an average accuracy of $99.17 \%$ on the detection of microbubbles on lumen and $91.67 \%$ on the classification of pre-injection frames as having no microbubbles, with an average percentage of support vectors less than $1 \%$ of the total training samples. For the second approach, we obtained an average accuracy of $89.65 \%$ on the detection of baseline IVUS data and $96.78 \%$ on the classification of microbubbles as change, with an average percentage of support vectors less than $10 \%$ of the total number of samples used for training. Figure 2 depicts examples of the classification results on frames before injection and during injection 


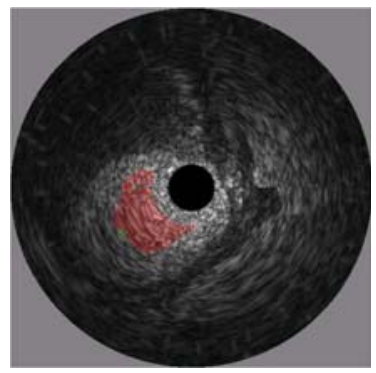

(a)

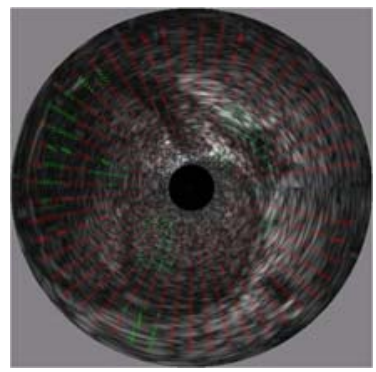

(c)

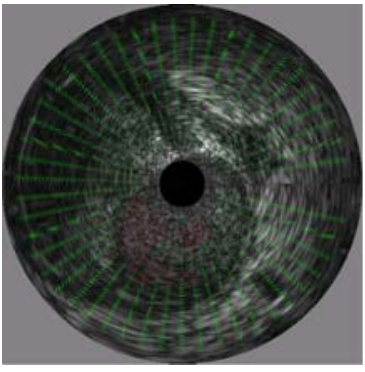

(b)

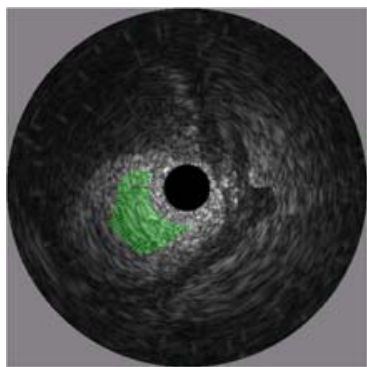

(d)

Fig. 2. Classification results in (a,d) a frame with microbubbles in the lumen and $(b, c)$ an IVUS frame before injection. For the first approach (a) and (b), the red color indicates the pixels classified as microbubbles and the green color those classified as non-microbubbles. For the second approach (c) and (d), the red color indicates the pixels classified as baseline IVUS and the green color those classified as an anomaly.

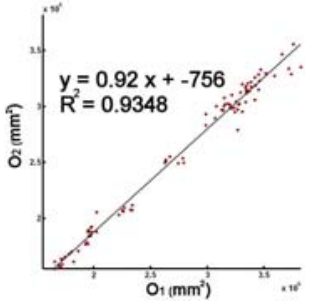

(a)

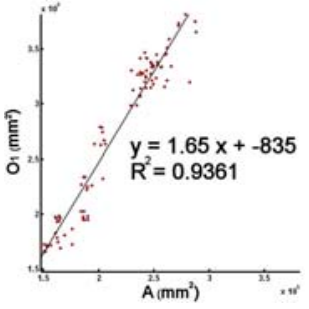

(b)

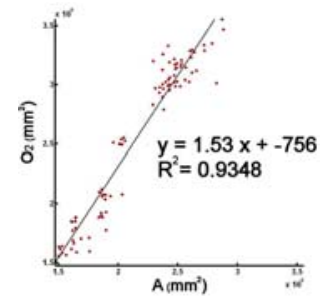

(c)

Fig. 3. Linear regression plot for (a) $\mathrm{O}_{1}$ vs $\mathrm{O}_{2}$, (b) A vs. $\mathrm{O}_{1}$ and (c) A vs. $\mathrm{O}_{2}$. Each point corresponds to one of the 90 segmented frames. 

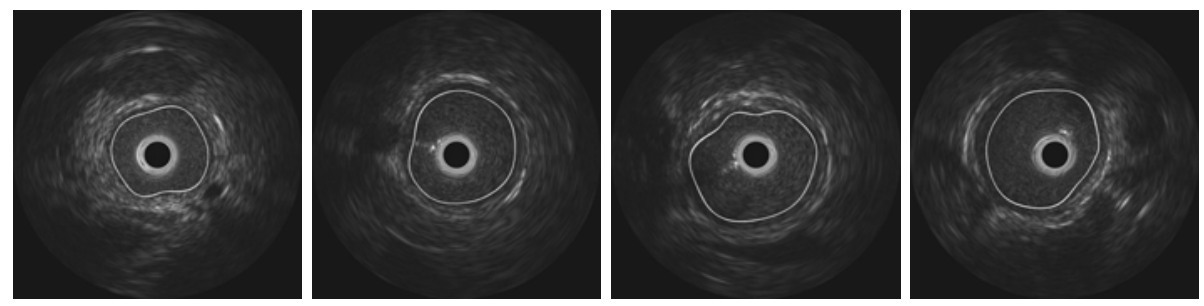

Fig. 4. Examples of segmentation results

for both approaches. One of the advantages of this methodology is that by using one-class learning, we did not need to provide "background" samples for building the models. In our case this was important because, although samples for microbubbles in lumen can be easily acquired by manual annotations from an expert, the background can consist of a wide variety of other imaged tissues. Thus, obtaining samples for the other tissues may be difficult and laborintensive.

We test our RF-based segmentation method on 90 frames from $40 \mathrm{MHz}$ sequences and the results were evaluated by comparing the agreement between areas corresponding to lumen on each frame by our method (A) with manual segmentations from two expert observers $\left(\mathrm{O}_{1}\right.$ and $\left.\mathrm{O}_{2}\right)$. The resulting mean Dice similarity coefficient was $s=90.27$. In addition, we performed linear regression. The coefficient of determination $\left(R^{2}\right.$, where $R$ is the linear correlation) for area differences between $\mathrm{O}_{1}$ and $\mathrm{O}_{2}\left(\mathrm{O}_{1}, \mathrm{O}_{2}\right)$ was $R^{2}=0.98$, and $R^{2}=0.93$ and $R^{2}=0.93$ for $\left(\mathrm{A}, \mathrm{O}_{1}\right)$ and $\left(\mathrm{A}, \mathrm{O}_{2}\right)$, respectively. Figure 3 depicts the results of this analysis and Fig. 4 depicts examples of the segmentation results.

\section{Conclusions}

We have presented methods for the analysis of IVUS data based on the RF signal. Future developments in VV detection methods will consist of using the scattering model to extract novel features to be used in combination with machine learning techniques for the detection of contrast agent within the plaque. The techniques presented in this paper may contribute significantly in the detection of neovascularization within atherosclerotic plaques. However, since VV is not the only cardiovascular risk biomarker, the combination of data from IVUS and other imaging modalities is necessary in order to provide an effective way to detect vulnerable patients. The expected impact of our work stems from the fact that sudden heart attack remains the number one cause of death in the US, and unpredicted heart attacks account for the majority of the $\$ 280$ billion burden of cardiovascular diseases. 


\section{References}

1. Naghavi, M., Libby, P., Falk, E., Casscells, S., Litovsky, S., Rumberger, J., Badimon, J., Stefanadis, C., Moreno, P., Pasterkamp, G., Fayad, Z., Stone, P., Waxman, S., Raggi, P., Madjid, M., Zarrabi, A., Burke, A., Yuan, C., Fitzgerald, P., Siscovick, D., de Korte, C., Aikawa, M., Airaksinen, K., Assmann, G., Becker, C., Chesebro, J., Farb, A., Galis, Z., Jackson, C., Jang, I., Koenig, W., Lodder, R., March, K., Demirovic, J., Navab, M., Priori, S., Rekhter, M., Bahr, R., Grundy, S., Mehran, R., Colombo, A., Boerwinkle, E., Ballantyne, C., Insull, J., Schwartz, W.R., Vogel, R., Serruys, P., Hansson, G., Faxon, D., Kaul, S., Drexler, H., Greenland, P., Muller, J., Virmani, R., Ridker, P., Zipes, D., Shah, P., Willerson, J.: From vulnerable plaque to vulnerable patient: A call for new definitions and risk assessment strategies: Part I. Circulation 108(14), 1664-1672 (2003)

2. Greenland, P., LaBree, L., Azen, S., Doherty, T., Detrano, R.: Coronary artery calcium score combined with Framingham score for risk prediction in asymptomatic individuals. Journal of the American Medical Association 291(2), 210-215 (2004)

3. Shaw, L., Raggi, P., Schisterman, E., Berman, D., Callister, T.: Prognostic value of cardiac risk factors and coronary artery calcium screening for all-cause mortality. Radiology 228(3), 826-833 (2003)

4. Taylor, A., Bindeman, J., Feuerstein, I., Cao, F., Brazaitis, M., O'Malley, P.: Coronary calcium independently predicts incident premature coronary heart disease over measured cardiovascular risk factors: Mean three-year outcomes in the Prospective Army Coronary Calcium (PACC) project. Journal of the American College of Cardiology 46(5), 807-814 (2005)

5. Wong, N.D., Budoff, M.J., Pio, J., Detrano, R.C.: Coronary calcium and cardiovascular event risk: Evaluation by age- and sex-specific quartiles. American Heart Journal 143(3), 456-459 (2002)

6. Gossl, M., Malyar, N., Rosol, M., Beighley, P., Ritman, E.: Impact of coronary vasa vasorum functional structure on coronary vessel wall perfusion distribution. American Journal of Physiology - Heart and Circulatory Physiology 285(5), H2019H2026 (2003)

7. Brunner, G., Chittajallu, D., Kurkure, U., Kakadiaris, I.: Toward the automatic detection of coronary artery regions in non-contrast Computed Tomography data. International Journal of Cardiovascular Imaging (in press, 2009)

8. Brunner, G., Chittajallu, D., Kurkure, U., Kakadiaris, I.: A heart-centered coordinate system for the detection of coronary artery zones in non-contrast Computed Tomography data. In: Proc. Medical Image Computing and Computer-Assisted Intervention Workshop on Computer Vision for Intravascular and Intracardiac Imaging, New York, NY, September 10 (2008)

9. Kurkure, U., Chittajallu, D., Brunner, G., Yalamanchili, R., Kakadiaris, I.: A supervised classification-based method for coronary calcium detection in non-contrast CT. International Journal of Cardiovascular Imaging (in press, 2009)

10. Kurkure, U., Chittajallu, D., Brunner, G., Yalamanchili, R., Kakadiaris, I.: Detection of coronary calcifications using supervised hierarchical classification. In: Proc. Medical Image Computing and Computer-Assisted Intervention Workshop on Computer Vision for Intravascular and Intracardiac Imaging, New York, NY, September 10 (2008)

11. Brunner, G., Kurkure, U., Chittajallu, D., Yalamanchili, R., Kakadiaris, I.: Toward unsupervised classification of calcified arterial lesions. In: Proc. 11th International Conference on Medical Image Computing and Computer Assisted Intervention, New York, NY, September 6-9, pp. 144-152 (2008) 
12. Chittajallu, D., Balanca, P., Kakadiaris, I.: Automatic delineation of the inner thoracic region in non-contrast CT data. In: Proc. 31st International Conference of the IEEE Engineering in Medicine and Biology Society, Minneapolis, MN, September 2-6 (2009)

13. Kurkure, U., Avila-Montes, O., Kakadiaris, I.: Automated segmentation of thoracic aorta in non-contrast CT images. In: Proc. IEEE International Symposium on Biomedical Imaging: From Nano to Macro, Paris, France, May 14-17 (2008)

14. Pednekar, A., Kurkure, U., Kakadiaris, I., Muthupillai, R., Flamm, S.: Left ventricular segmentation in MR using hierarchical multi-class multi-feature fuzzy connectedness. In: Proc. 7th International Conference on Medical Image Computing and Computer Assisted Intervention, Rennes, Saint-Malo, France, September 2630, pp. 402-410 (2004)

15. O'Malley, S., Vavuranakis, M., Naghavi, M., Kakadiaris, I.: Intravascular ultrasound-based imaging of vasa vasorum for the detection of vulnerable atherosclerotic plaque. In: Proc. Medical Image Computing and Computer-Assisted Intervention, Palm Springs, CA, October, pp. 343-351 (2005)

16. O’Malley, S., Naghavi, M., Kakadiaris, I.: One-class acoustic characterization applied to blood detection in IVUS. In: Proc. 10th International Conference on Medical Image Computing and Computer Assisted Intervention, Brisbane, Australia, October 29 - November 2, pp. 202-209 (2007)

17. Papaioannou, T., Vavuranakis, M., Androulakis, A., Lazaros, G., Kakadiaris, I., Vlaseros, I., Naghavi, M., Kallikazaros, I., Stefanadis, C.: In-vivo imaging of carotid plaque neoangiogenesis with contrast-enhanced harmonic ultrasound. International Journal of Cardiology 134(3), 110-112 (2009)

18. Vavuranakis, M., Kakadiaris, I., O'Malley, S., Papaioannou, T., Sanidas, E., Naghavi, M., Carlier, S., Tousoulis, D., Stefanadis, C.: A new method for assessment of plaque vulnerability based on vasa vasorum imaging, by using contrastenhanced intravascular ultrasound and automated differential image analysis. International Journal of Cardiology 130, 23-29 (2008)

19. Vavuranakis, M., Kakadiaris, I., O'Malley, S., Papaioannou, T., Carlier, S., Naghavi, M., Stefanadis, C.: Contrast-enhanced intravascular ultrasound: Combining morphology with activity-based assessment of plaque vulnerability. Expert Review of Cardiovascular Therapy 5, 915-917 (2007)

20. Vavuranakis, M., Papaioannou, T., Kakadiaris, I., O’Malley, S., Naghavi, M., Filis, K., Sanidas, E., Papalois, A., Stamatopoulos, I., Stefanadis, C.: Detection of perivascular blood flow in vivo by contrast-enhanced intracoronary ultrasonography and image analysis: An animal study. Clinical and Experimental Pharmacology and Physiology 34(12), 1319-1323 (2007)

21. Mendizabal-Ruiz, E., Kakadiaris, I.: One-class acoustic characterization applied to contrast agent detection in IVUS. In: Proc. Medical Image Computing and Computer-Assisted Intervention Workshop on Computer Vision for Intravascular and Intracardiac Imaging, New York, NY, September 10 (2008)

22. Fontaine, I., Bertrand, M., Cloutier, G.: A system-based approach to modeling the ultrasound signal backscattered by red blood cells. Biophysical Journal 77(5), 2387-2399 (1999)

23. Mendizabal-Ruiz, E., Biros, G., Kakadiaris, I.: An inverse scattering algorithm for the segmentation of the luminal border on intravascular ultrasound data. In: Proc. 12th International Conference on Medical Image Computing and Computer Assisted Intervention, London, UK, September 20-24 (2009) 